\title{
Efficacité comparée des systèmes d'évaluation énergétique des aliments pour la prévision des performances de croissance du porc
}

\author{
JM Perez ${ }^{1 *, \star \star}$, Y Henry 1, D Sauvant 2, L Jacquot 3, V Ricca 3 \\ avec la collaboration technique de G Conseil, M Lemarié, $Y$ Lebreton, $L$ Jaffrennou, \\ N Mézière, Y Colléaux pour l'INRA; de N Mandran pour l'INA Paris-Grignon \\ et de D Lebas pour Sanders Aliments \\ 1 INRA, station de recherches porcines, centre de Rennes, 35590 Saint-Gilles; \\ 2 INA Paris-Grignon, département des sciences animales, \\ 16, rue Claude-Bernard, F 75231 Paris Cedex 05; \\ ${ }^{3}$ Sanders Aliments, 17, quai de I'Industrie, F 91201 Athis-Mons Cedex, France
}

(Reçu le 5 juin 1990; accepté le 4 septembre 1990)

Résumé - Cette étude avait pour objectif de comparer l'efficacité de 2 systèmes d'évaluation énergétique des aliments (énergie digestible : $E D$; énergie nette engraissement : $E N E$ ) pour la prévision des performances de production du porc. Afin d'explorer la plage de variation simultanée la plus large possible des valeurs $E D$ et $E N E$ formulables, 8 régimes ont été testés selon un dispositif factoriel $2 \times 4$ :

-2 types de régimes «impairs» $(1,3,5,7)$ et «pairs» $(2,4,6,8)$ reflétant respectivement la formulation dans les systèmes $E D$ et $E N E$;

- 4 densités énergétiques croissantes suivant une progression concomitante dans les 2 systèmes et correspondant aux couples de régimes $1-2,3-4,5-6$ et $7-8$.

Par rapport aux régimes impairs essentiellement à base de blé et de tourteau de soja, les régimes pairs, à base d'orge et de produits de substitution, se caractérisent par des teneurs réduites en protéines et plus élevées en matières grasses. Les contraintes de formulation et les plans de rationnement étaient ajustés de manière à assurer les mêmes apports journaliers d'énergie selon le système, dans chacun des groupes de régimes impairs et pairs, et les mêmes apports journaliers de lysine dans les 8 régimes.

L'étude a donné lieu à 2 expériences en lots (essais 1 et 2), utilisant un nombre égal de femelles et de mâles castrés par essai, complétées par une expérience de digestibilité (4 animaux par régime) pour contrôler la valeur énergétique des aliments, dans les systèmes considérés. L'essai 1 a été réalisé sur 160 porcs de race Large White en loges individuelles entre 25 et $93 \mathrm{~kg}$ de poids vif à raison de 20 animaux par régime. L'essai 2, qui portait sur 6 régimes (3-8), a été conduit en loges collectives sur 336 porcs croisés entre 23 et $98 \mathrm{~kg}$ de poids vif, à raison de 4 groupes de 14 animaux par régime.

Les régimes pairs, principalement aux plus faibles densités énergétiques, ont procuré pendant la première phase de la croissance des performances plus faibles que celles obtenues avec les régimes impairs en raison d'un déficit en acides aminés limitants secondaires (thréonine, méthionine), accentué par une digestibilité réduite des matières azotées. Inversement, pendant la phase de finition et sur l'ensemble de la période d'engraissement, ce sont les régimes pairs aux densités énergé-

\footnotetext{
* Correspondance et tirés à part.

** Adresse actuelle: INRA, laboratoire de recherches sur l'élevage du lapin, centre de recherches de Toulouse, Auzeville, BP 27, 31326 Castanet-Tolosan Cedex, France.
} 
tiques les plus élevées (régimes 6 et 8 ) qui ont permis d'obtenir les meilleures performances (gain pondéral, indice de consommation, pourcentage et gain de muscle et de gras).

Les variations d'indice de consommation énergétique, exprimé en Mcal $/ \mathrm{kg}$ de gain de poids vif total ou vide, semblent globalement moins dépendantes des fluctuations de composition des régimes (type, densité) dans le système ED que dans le système ENE. De même, la prévision des dépôts tissulaires et de l'énergie fixée à partir de l'énergie ingérée semble plus précise dans le système $E D$ que dans le système ENE. Le nouveau système d'évaluation de l'énergie nette proposé par l'INRA "énergie nette porc" ou EN porc) a permis encore d'accroître le degré d'indépendance de l'indice de consommation énergétique en fonction des modalités nutritionnelles, ainsi que la précision de la prévision des variations des dépôts de muscle et de gras.

porc / valeur énergétique des aliments / système énergétique / énergie digestible / énergie nette / croissance

Summary - The efficacy of feed energy evaluation systems for predicting growth performance in pigs. The efficacy of 2 feed energy evaluation systems (digestible energy: $D E$, net energy for fattening: NEF) for predicting growth performance (feed efficiency, daily muscle and fat gain) in the pig was measured by comparing 8 diets obtained from formulation in both systems and arranged according to a $2 \times 4$ factorial plan:

-2 types of diets, designated "uneven" $(1,3,5,7)$ and "even" $(2,4,6,8)$, respectively, differing by ingredient origin and chemical composition;

- 4 increasing energy density levels, according to a simultaneous progression in both systems and corresponding to diet pairs $1-2,3-4,5-6$ and 7-8.

Compared to the uneven diets, which were essentially based on wheat and soybean meal, the even diets which were obtained through formulation in NEF system had lower protein content, in conjunction with supplementary addition of industrial lysine, higher fat content, and were more diversified, with higher inclusion of fibrous by-products (wheat bran, beet pulp, maize gluten feed) in addition to barley as the cereal base of the diets. Formulation constraints (lysine-energy ratio, as the first limiting amino acid) and feeding scales were adjusted to maintain the same daily supply of energy and lysine between diets.

Two trials, with an equal number of females and castrated males, were conducted. The first (trial 1) involved 160 Large White pigs raised in individual pens between 25 and $93 \mathrm{~kg}$ live weight, with 20 animals per diet. In the second trial (trial 2) which was conducted in groups the number of experimental diets was restricted to 6 (diets 3 to 8). Three hundred thirty six crossbred pigs were used between 23 and $98 \mathrm{~kg}$ live weight, with 4 groups of 14 animals per diet. DE value and digestible nutrient contents were measured in a digestibility trial including 4 castrated male pigs of the Large White breed per diet.

During the first phase of the experimental period $(23-60 \mathrm{~kg}$ live weight interval in trial 1 and the period following the first $70 \mathrm{~d}$ in trial 2), lower performance (average daily gain, feed conversion ratio) were obtained with the even diets, especially at the lowest energy densities (diets 2 and 4), than with the uneven diets. This difference was due to lower levels of secondary limiting amino acids (threonine, methionine) in the even diets in combination with lower protein digestibility. On the contrary, during the finishing phase and the overall experimental period, the best growth performance (average daily gain, feed conversion ratio, percentage and daily rate of muscle and fat deposit) were obtained with the even diets at the highest energy densities (diets 6 and 8).

The effects of dietary factors (type of diet, energy density and interaction) on feed conversion ratios expressed in energy value (Mcal per $\mathrm{kg}$ total or empty live weight gain) in both systems were less apparent with DE compared to NEF. In the same manner, the amounts of muscle and fat gain or of total retained energy were better predicted with DE than with NEF. The new system for net energy evaluation for growth (EN porc) introduced by INRA was included in the comparison with DE and NEF. The results showed its efficacy in reflecting the expected independence of feed energy conversion values with changes in type of diet and energy density, as well as in predicting tissue deposition and energy gain from the computed daily mean energy intake in the different systems.

plg / feed energy value / feed energy system / digestible energy / net energy / growth 


\section{INTRODUCTION}

De nombreux systèmes, différant par leur concept et les modalités d'utilisation, ont été proposés pour estimer la valeur énergétique des aliments destinés au porc. Leurs avantages et inconvénients respectifs ont été analysés en détail dans des revues récentes (Henry et Perez, 1982, 1983; Henry et al, 1988). Néanmoins, les conséquences techniques et économiques du choix de tel ou tel système ont été insuffisamment mesurées, selon que l'on s'intéresse à la formulation de l'aliment ou à sa valorisation par l'animal.

Un des éléments du choix d'une unité d'alimentation énergátique concerne l'appréciation de la hiérarchie réelle des valeurs des matières premières. En effet, comme on a pu le vérifier dans une étude initiale (Brette et al, 1986), les modifications de leurs valeurs énergétiques relatives suivant les systèmes influencent fortement le taux d'incorporation des composants des mélanges alimentaires formulés au moindre coût. En outre, la fiabilité d'un système énergétique doit être appréciée par la précision de la relation entre les apports alimentaires (énergétiques et azotés) et les performances de production (gain journalier de tissus maigres et gras, efficacité alimentaire). Sur ce dernier point, les travaux réalisés sont quasiment inexistants. Seuls Borggreve et al (1975), et plus récemment Jongbloed et al (1986), ont testé la fiabilité du système "énergie nette engraissement" (Schiemann et al, 1971) pour la prévision des performances.

L'objectif de la présente étude est donc de comparer les performances zootechniques obtenues à partir d'une série de régimes représentatifs des formules optimisées dans chacun des systèmes "énergie digestible» $(E D)$ et "énergie nette engrais- sement" (ENE) de manière à pouvoir en dissocier les effets. En outre, nous avons tenu compte des travaux récents de l'INRA sur le sujet en introduisant dans la comparaison le nouveau système "énergie nette porc" (EN porc) (Noblet et al, 1989). Cette étude a fait l'objet d'une collaboration entre I'INRA (station de recherches porcines, Saint-Gilles), I'INA-PG (département des sciences animales) et la firme Sanders, dans le cadre de l'action concertée "Protéines en alimentation animale" du ministère de la Recherche et de la Technologie (MRT).

\section{MATÉRIEL ET MÉTHODES}

L'étude a donné lieu à 2 expériences en lots, réalisées respectivement dans les installations experimentales de I'INRA Saint-Gilles (essai 1) et Sanders-Sourches (essai 2), et complétées par un essai de digestibilité conduit à l'INRA, pour contrôler la valeur énergétique des régimes expérimentaux dans les systèmes considérés.

\section{Choix et composition des régimes expérimentaux}

Un fichier préalable des caractéristiques d'une trentaine de matières premières a été constitué essentiellement à partir des données de composition chimique et de valeur nutritive (teneur en $E D$ ) des tables INRA (1984) et des coefficients de digestibilité des tables hollandaises (CVB, 1983). La teneur en ENE a été estimée à partir des éléments digestibles à l'aide de l'équation initiale de Nehring et al (1969) retenue dans les tables hollandaises (tableau I).

A partir d'un ensemble de 90 mélanges formulés par programmation linéaire (Brette et al, 1986), nous avons cherché à concevoir des régimes de même teneur en énergie digestible présentant des valeurs très différentes en énergie nette engraissement et réciproquement. Huit régimes ont été retenus dans le but d'explorer la plage de variation simultanée la plus large pos- 


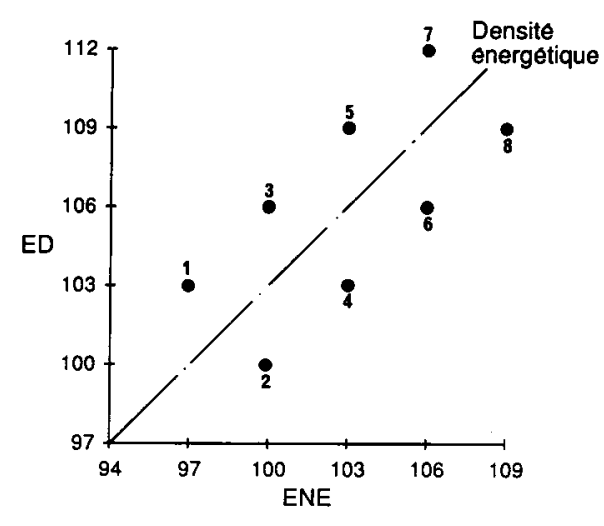

Fig 1. Schéma expérimental.

sible des valeurs $E D$ et $E N E$ formulables. Le schéma expérimental (fig 1) correspond à une progression concomitante par couple (1-2, 3-4, $5-6,7-8$ ) de la densité énergétique dans chacun des 2 systèmes. Cela permet de définir 2 groupes de régimes «impairs", avec $E N=a_{1}$ $E D+b_{1}$ et "pairs", avec $E N=a_{2} E D+b_{2}$ (tableau I), se distinguant par la nature de la céréale (respectivement blé et orge), le taux de protéines (plus faible dans les régimes upairs") et la complexité de la formule (plus grande dans les régimes "pairs"). Le dispositif expérimental considéré est ainsi de type factoriel $2 \times 2 \times 4$, correspondant aux facteurs "sexe" (2 niveaux), atype de régime" (2 niveaux) et "densité énergétique " (4 niveaux).

L'apport azoté a été défini en ajustant la teneur en lysine totale en fonction de la densité énergétique $(0,81 \%$ pour la densité 100 dans les régimes 1 et 2). Ceci a nécessité la supplémentation des régimes "pairs" par de la lysine industrielle.

La composition centésimale et les caractéristiques théoriques des régimes expérimentaux sont présentées dans le tableau I. La fabrication des aliments a été réalisée pour l'ensemble des essais dans l'usine Sanders (Le Mans) à partir des mêmes lots de matières premières.

\section{Étude de digestibilité}

Trente-deux porcs mâles castrés de race Large White sont placés en cages spécialement équi- pées pour les bilans digestifs à raison de 4 animaux par régime, selon les modalités déjà décrites (Perez et al, 1984). Après une période d'adaptation de $7 \mathrm{j}$ aux régimes expérimentaux, ils sont soumis à une période de collecte totale des fèces et de l'urine pendant $10 \mathrm{j}$ consécutifs, à un poids vif moyen de $42 \mathrm{~kg}$. Au cours de cette période, les niveaux alimentaires d'énergie sont maintenus constants $(0,3 \mathrm{Mcal} E D$ calculée $/ \mathrm{kg}^{0,75}$ ). Les animaux reçoivent leur ration sous forme de granulés secs à raison de 2 repas par jour et disposent d'eau à volonté.

\section{Expérimentations sur animaux en lots}

\section{Conception des plans d'alimentation}

Dans les 2 essais en lots les plans d'alimentation ont été définis en fonction d'une densité énergétique moyenne qui tient compte des variations simultanées des concentrations en ED et ENE (valeur 100 pour le régime 2). Comme l'indiquent la figure 1 et le tableau II, cela se traduit par une progression de la densité énergétique moyenne par paliers successifs regroupant les couples de régimes 1-2 (100), 3-4 (103), 5-6 (106) et 7-8 (109). En conséquence, les niveaux alimentaires varient en raison inverse des densités énergétiques ainsi définies, selon un plan de rationnement de référence préalablement fixé pour les régimes 5 et 6 (tableau III). Les mâles castrés et les femelles sont soumis au même plan de rationnement ajusté en fonction du poids vif et reçoivent un régime unique pendant la totalité de l'expérience.

\section{Essai 1-INRA}

Un effectif de 160 porcs ( 80 mâles castrés et 80 femelles) de race Large White provenant du troupeau expérimental de I'INRA Saint-Gilles, d'un poids vif moyen initial de $25,4 \mathrm{~kg}(s=1,7)$, est réparti en 8 lots selon la méthode des blocs en tenant compte du sexe, du poids et de l'âge au départ de l'expérience. Ils sont élevés en loges individuelles sur sol bétonné avec litière de paille dans un bâtiment fermé jusqu'à un poids final moyen d'abattage de $93,3 \mathrm{~kg}$ ( $s=$ $1,9)$. 
Tableau I. Composition centésimale et caractéristiques théoriques des régimes.

\begin{tabular}{|c|c|c|c|c|c|c|c|c|}
\hline Régimes & 1 & 2 & 3 & 4 & 5 & 6 & 7 & 8 \\
\hline Blé & 40,3 & - & 43,5 & - & 51 & - & 60,1 & - \\
\hline Orge & - & 48,5 & - & 54,2 & - & 49,81 & - & 51,85 \\
\hline Son de blé & 15 & 15 & 15 & 15 & 6,5 & 15 & - & 8,6 \\
\hline Corn gluten feed & 1,5 & 7,3 & - & - & - & - & - & - \\
\hline Pulpes betteraves & 5 & 5 & 0,9 & 2,8 & - & - & - & - \\
\hline Mélasse & 8 & 8 & 8 & 8 & 8 & 8 & 3,9 & 8 \\
\hline Pois & 15 & - & 15 & 2,6 & 15 & 10,2 & 15 & 13,4 \\
\hline T colza & 5 & 5 & 5 & 5 & 5 & 5 & 5 & 5 \\
\hline T soja 48 & 7,6 & 1,5 & 9,1 & 1,9 & 10,7 & 0,6 & 12,1 & 1,7 \\
\hline$F$ viande $55 G$ & - & 5 & - & 5 & - & 5 & - & 5 \\
\hline Graisses animales & 0,2 & 2,8 & 1 & 3,5 & 1,2 & 4,4 & 1,2 & 4,9 \\
\hline L-Lysine $\mathrm{HCl}^{1}$ & - & 0,2 & - & 0,2 & - & 0,19 & - & 0,15 \\
\hline Carbonate calcium & 0,9 & 0,7 & 1 & 0,8 & 0,8 & 0,8 & 0,7 & 0,4 \\
\hline Phosphate bicalcique & 0,5 & - & 0,5 & - & 0,8 & - & 1 & - \\
\hline Minéraux, vitamines ${ }^{2}$ & 1 & 1 & 1 & 1 & 1 & 1 & 1 & 1 \\
\hline Matières azotées (\%) & 16,3 & 14,7 & 16,7 & 14,3 & 16,9 & 14,6 & 17,5 & 15,0 \\
\hline Lysine (\%) & 0,81 & 0,81 & 0,83 & 0,83 & 0,85 & 0,85 & 0,88 & 0,87 \\
\hline$E D t, \mathrm{kcal} / \mathrm{kg}^{3}$ & 3000 & 2910 & 3090 & 3000 & 3180 & 3090 & 3265 & 3180 \\
\hline ENEt, $\mathrm{kcal} / \mathrm{kg}^{4}$ & 2037 & 2100 & 2100 & 2163 & 2163 & 2226 & 2226 & 2289 \\
\hline ENEtED $t^{5}$ & 0,68 & 0,72 & 0,68 & 0,72 & 0,68 & 0,72 & 0,68 & 0,72 \\
\hline
\end{tabular}

1 Lysine monochiorhydrate à $79 \%$ de pureté. 2 Dont $0,5 \%$ de sel. ${ }^{3}$ Énergie digestible des régimes calculée à partir des valeurs des matières premières des tables INRA. ${ }^{4}$ Énergie nette engraissement (Nehring ot al, 1969): ENE = 2,59 MAD + 8,63 MGD + 1,50 CBD + 3,03 ENAD; éléments digestibles en g/kg déduits des tables. ${ }^{5}$ Régimes impairs : $E N E t=-99+0,71 E D t$ régimes pairs : $E N E t=-63+0,70 E D t$, soit un écart de $162 \mathrm{kcal} E N E$ entre les 2 groupes de régimes, pour une même valeur $E D$.

Les aliments sont distribués sous forme de granulés une seule fois par jour. Les quantités consommées sont enregistrées quotidiennement et les plans d'alimentation ajustés après pesée hebdomadaire des animaux. L'eau est fournie à volonté à l'aide d'un abreuvoir automatique.

Tableau II. Valeurs énergétiques relatives théoriques et niveaux d'alimentation.

\begin{tabular}{|c|c|c|c|c|c|c|c|c|}
\hline Régimes & 1 & 2 & 3 & 4 & 5 & 6 & 7 & 8 \\
\hline $\begin{array}{l}E D f^{1} \\
E N E t^{1}\end{array}$ & $\begin{array}{r}103 \\
97\end{array}$ & $\begin{array}{l}100 \\
100\end{array}$ & $\begin{array}{l}106 \\
100\end{array}$ & $\begin{array}{l}103 \\
103\end{array}$ & $\begin{array}{l}109 \\
103\end{array}$ & $\begin{array}{l}106 \\
106\end{array}$ & $\begin{array}{l}112 \\
106\end{array}$ & $\begin{array}{l}109 \\
109\end{array}$ \\
\hline $\begin{array}{l}\text { Densité énergétique moyenne } \\
\text { Niveau d'alimentation }\end{array}$ & \multicolumn{2}{|c|}{$\begin{array}{l}100 \\
106\end{array}$} & \multicolumn{2}{|c|}{$\begin{array}{l}103 \\
103\end{array}$} & \multicolumn{2}{|c|}{$\begin{array}{l}106 \\
100\end{array}$} & \multicolumn{2}{|c|}{$\begin{array}{r}109 \\
97\end{array}$} \\
\hline
\end{tabular}

1 Cf Tableau !. 
Tableau III. Plans d'alimentation'.

\begin{tabular}{cccc}
\hline $\begin{array}{c}\text { Poids vif } \\
(\mathrm{kg})\end{array}$ & $\begin{array}{c}\text { Aliment } \\
(\mathrm{kg} / \mathrm{j})\end{array}$ & $\begin{array}{c}\text { Poids vif } \\
(\mathrm{kg})\end{array}$ & $\begin{array}{c}\text { Aliment } \\
(\mathrm{kg} / \mathrm{j})\end{array}$ \\
\hline & & & \\
20 & 0,98 & 60 & 2,35 \\
25 & 1,22 & 65 & 2,47 \\
30 & 1,46 & 70 & 2,58 \\
35 & 1,62 & 75 & 2,65 \\
40 & 1,78 & 80 & 2,72 \\
45 & 1,93 & 85 & 2,77 \\
50 & 2,07 & 90 & 2,82 \\
55 & 2,21 & & \\
\hline
\end{tabular}

${ }^{1}$ Base 100 pour les régimes 5 et 6 .

Les porcs sont abattus après un jeûne de $16 \mathrm{~h}$. Les mesures suivantes sont effectuées :

- poids net de la carcasse chaude;

- poids des viscères et des contenus digestifs;

- longueur de la carcasse, totale et restreinte;

- mesures d'épaisseurs de gras et de muscles

à l'aide de l'appareil Fat-O-Meater (FOM);

- découpe parisienne normalisée (DPN) sur la totalité des carcasses;

- dissection partielle du jambon (gras externe, os, peau, muscles non triés) sur 5 femelles et 5 mâles castrés par régime.

Les teneurs en muscle et en gras sont calculées à partir des mesures FOM et DPN, selon Desmoulin et al (1988). Les gains journaliers de muscle et de gras sont estimés de la même façon à partir des quantités finales de ces tissus dans la carcasse et des quantités initiales calculées en fonction du poids vif et selon le sexe, à partir d'équations de prédiction établies pour des animaux de même race et provenant du même troupeau expérimental (Noblet et $\mathrm{Ka}$ rege, communication personnelle).

\section{Essai 2-Sanders}

La comparaison est limitée à 6 régimes $(3-8)$ correspondant aux 3 niveaux supérieurs de densité énergétique. L'essai est réalisé sur 336 porcs (moitié femelles, moitié mâles castrés), d'une souche commerciale de type croisé
(France-Hybrides), à partir d'un poids vif moyen de $23 \mathrm{~kg}(s=2,7)$, à raison de 2 répétitions de 14 animaux de chaque sexe par traitement. Dans chacune des répétitions, les 14 porcs de même sexe sont élevés collectivement dans une même loge et alimentés au sol. Les aliments sont distribués sous forme de granulés, 2 fois par jour, avec l'eau à volonté.

Au cours de l'essai, les porcs sont pesés globalement par case tous les $14 j$ afin de suivre le plan de rationnement. Ils sont pesés individuellement au début de l'essai, après $70 \mathrm{j}$, et au stade final d'abattage, après 98,112 ou $126 \mathrm{j}$. Les mesures de consommation d'aliment et les calculs correspondants d'indice de consommation sont réalisés globalement au niveau de la loge.

A l'abattage, au poids vif moyen de $96,9 \mathrm{~kg}$ $(s=8,7)$, on a procédé aux mesures du rendement à l'abattage (à chaud et à froid, après un ressuyage de $24 \mathrm{~h}$ ), d'épaisseur de lard au dos et au rein, complétées par la mesure de la teneur en muscle à l'aide de l'appareil Fat-OMeater sur 183 carcasses (en moyenne 30 par traitement). En outre, lors de la deuxième série d'abattage, une découpe parisienne normalisée des carcasses entières a été effectuée sur 130 porcs, dont 78 femelles et 62 mâles castrés ayant atteint un poids proche de $100 \mathrm{~kg}$ après $112 \mathrm{j}$.

\section{Méthodes d'analyse}

La composition chimique des régimes est contrôlée par les méthodes classiques (cendres, azote, énergie brute, cellulose Weende). En outre, les matières grasses sont dosées après hydrolyse acide et extraction à l'éther diéthylique à l'aide d'un appareil semi-automatique de type Soxtec. Les teneurs en constituants pariétaux (ADF, NDF, AD lignine: $A D L$ ) sont déterminées selon les méthodes de Van Soest (1963), Van Soest et Wine (1967), adaptées par Giger et al (1979). L'amidon a été dosé par la méthode polarimétrique (Ewers) et les sucres totaux déterminés par la technique de LuffSchoorl. Les acides aminés sont dosés par chromatographie liquide sur colonnes échangeuses d'ions. Sur les échantillons de fèces lyophilisés, on a déterminé pour chaque animal les teneurs en cendres, azote, cellulose Weende, 
fractions Van Soest, matières grasses et énergie brute. Sur les échantillons d'urine, on a procédé au dosage de l'azote et, après lyophilisation, à la mesure du contenu en énergie brute.

\section{Analyse statistique}

Les données expérimentales sont traitées par les techniques d'analyse de variance et de covariance, selon la procédure GLM du logiciel SAS (1988), complétée par la procédure REG pour l'analyse de régression. Dans le cas de données manquantes, les résultats par traitement sont exprimés sous forme de moyennes ajustées. Les analyses de variance et de covariance sont réalisées en prenant en compte, outre les effets blocs (essai 1) ou répétitions (essai 2), les effets principaux du dispositif factoriel (sexe, type de régime, densité énergétique) et les effets d'interaction (type $x$ densité, type $x$ sexe et densité $x$ sexe). Un traitement d'ensemble des données des 2 essais a porté sur les résultats moyens par traitement et par sexe.

\section{RÉSULTATS}

\section{Analyse des régimes (tableau IV)}

Les résultats moyens d'analyse des régimes sont rapportés dans le tableau IV. Comparativement aux valeurs théoriques du protocole, ils font apparaître pour les protéines des valeurs supérieures aux prévisions pour les régimes pairs à base d'orge. Ces différences proviennent de teneurs plus élevées en protéines des orges récoltées en 1984 par rapport aux valeurs des tables INRA retenues pour la formulation $(11,9$ contre $10 \%)$. Les teneurs en lysine des régimes sont conformes aux valeurs théoriques. En revanche, pour la thréonine et les acides aminés soufrés, les valeurs mesurées se révèlent légèrement inférieures à celles de la formulation, ce qui conduit, par rapport aux recommanda- tions, à des teneurs suboptimales dans les régimes pairs.

\section{Résultats de digestibilité (tableau V)}

L'examen des résultats de digestibilité de la matière organique, de l'énergie et des protéines fait apparaître une différence systématique entre les régimes impairs (à base de blé) et les régimes pairs (à base d'orge), ainsi qu'un effet de la densité énergétique (1-2 vs $3-4$ vs $5-6$ vs $7-8$ ). Les CUD moyens de l'énergie et de l'azote sont plus élevés (+ 8 points) dans les régimes impairs que dans les régimes pairs : 85,1 et 85,6 contre 77,2 et 77,3 respectivement. Dans les 2 cas, les CUD augmentent avec la densité énergétique théorique du régime en relation inverse avec la teneur en parois. Pour ces dernières, et quel que soit le critère retenu, la digestibilité est toujours plus faible dans les régimes pairs à base d'orge $(36,2$ contre $48,1 \%$ pour le résidu NDF par exemple). Mis à part ce phénomène, la digestibilité des constituants des parois n'est pas affectée par leurs taux correspondants dans les régimes.

Les CUD apparents des matières grasses (calculés après hydrolyse acide des excreta fécaux) sont plus faibles que les valeurs théoriques déduites des tables hollandaises. Ces dernières surestiment donc fortement la digestibilité des lipides même si l'on tient compte de la part de l'endogène surtout importante dans les régimes peu pourvus en graisses ajoutées. Les valeurs des CUD apparents des matières grasses sont très voisines, bien qu'un peu plus élevées dans les régimes pairs qui bénéficient d'une incorporation plus forte de graisses animales : en moyenne 65,1 contre 63,1 dans les régimes impairs. L'analyse de régression des quantités digestibles sur les quantités 
Tableau IV. Résultats d'analyse des régimes.

\begin{tabular}{lrrrrrrrr}
\hline \multicolumn{1}{c}{ Régimes } & \multicolumn{1}{c}{ 1 } & \multicolumn{1}{c}{3} & \multicolumn{1}{c}{4} & \multicolumn{1}{c}{5} & 6 & 7 & 8 \\
\hline & & & & & & & & \\
Matière sèche ${ }^{1}$ & 87,5 & 86,9 & 87,5 & 87,2 & 87,5 & 87,5 & 87,4 & 87,5 \\
Matière organique & 81,8 & 80,3 & 81,9 & 81,0 & 81,8 & 81,5 & 82,2 & 81,7 \\
Matières azotées & 16,8 & 16,0 & 16,8 & 16,2 & 16,9 & 16,4 & 17,1 & 16,7 \\
Matières grasses & 2,4 & 6,2 & 3,3 & 6,7 & 3,0 & 7,3 & 2,8 & 7,3 \\
Cellulose brute & 4,5 & 4,8 & 4,0 & 4,7 & 3,2 & 4,3 & 3,2 & 4,1 \\
NDF & 12,7 & 16,6 & 13,1 & 13,9 & 9,3 & 13,2 & 8,8 & 13,1 \\
ADF & 6,6 & 7,0 & 5,9 & 6,1 & 4,9 & 5,6 & 4,6 & 5,7 \\
ADL & 1,4 & 1,5 & 1,4 & 1,6 & 1,2 & 1,4 & 0,9 & 1,4 \\
Amidon & 36,0 & 28,4 & 36,5 & 31,9 & 38,3 & 32,4 & 41,9 & 32,1 \\
Sucres totaux & 6,4 & 9,4 & 7,1 & 6,9 & 7,1 & 6,9 & 6,2 & 7,6 \\
Lysine & 0,80 & 0,80 & 0,82 & 0,83 & 0,85 & 0,86 & 0,89 & 0,88 \\
Thréonine & 0,54 & 0,53 & 0,56 & 0,51 & 0,56 & 0,50 & 0,58 & 0,48 \\
Méthionine & 0,24 & 0,22 & 0,25 & 0,21 & 0,25 & 0,21 & 0,26 & 0,21 \\
Méthionine + cystine & 0,55 & 0,51 & 0,59 & 0,48 & 0,60 & 0,48 & 0,63 & 0,49 \\
EB ${ }^{2}$ & 3717 & 3913 & 3760 & 3986 & 3751 & 4059 & 3837 & 4048 \\
\hline
\end{tabular}

${ }^{1}$ Caractéristiques analytiques exprimées en pourcentage du produit brut. ${ }^{2}$ Énergie brute en $\mathrm{kcal} / \mathrm{kg}$.

ingérées aboutit à une estimation de la digestibilité des matières grasses ajoutées de $67 \%(s=2,6 ; r=0,995)$.

En ce qui concerne l'estimation des valeurs énergétiques ( $E D, E M)$, la précision des mesures est correcte, puisque les erreurs types oscillent entre $3-40 \mathrm{kcal}$ selon les régimes, ce qui correspond à un coefficient moyen de variation de $1,4 \%$. La valeur $E D$ est plus faible en moyenne dans les régimes à base d'orge $(-3,6 \%)$, alors que leur contenu en énergie brute est accru de $6,2 \%$ en raison de l'incorporation de graisses. Le rapport EM/ED est supérieur dans les régimes pairs $(95,6$ contre $95,0 \%$ ) en raison d'une teneur un peu plus faible en protéines $(-0,6$ point). Dans l'ensemble, les valeurs $E M$ sont très fortement corrélées avec les valeurs $E D$ $(r=0,99)$.

Comme le montre la figure 2, les valeurs $E D$ mesurées $\left(E D_{m}\right)$ sont bien corrélées $(r=0,96, E T R=1,2 \%)$ avec les va- leurs additives de formulation calculées à partir des données des tables INRA (1984) sur les matières premières (EDt). La hiérarchie entre les régimes est respectée à l'exception toutefois du régime 8 , dont la valeur $E D$ mesurée est inférieure de $2,4 \%$ en valeur relative à celle prévue au protocole. La précision de l'estimation ne peut être mise en cause pour ce régime, car sa teneur en énergie brute est strictement identique à la valeur théorique (calculée par régression à partir de sa composition chimique) et le coefficient de variation de la mesure du CUD de l'énergie est en l'occurrence inférieur à $0,2 \%$. Comparativement aux autres régimes pairs, l'écart relatif du régime 8 avec $\mathrm{a}$ valeur théorique pourrait s'expliquer à la fois par une proportion moindre de son de blé, dont la valeur énergétique aurait pu être sousestimée $(1,8$ point de cellulose brute en moins par rapport aux tables) et par une proportion plus élevée de graisses ajoutées, dont la valeur énergétique serait au 
Tableau V. Résultats de digestibilité.

\begin{tabular}{|c|c|c|c|c|c|c|c|c|}
\hline Régimes & 1 & 2 & 3 & 4 & 5 & 6 & 7 & 8 \\
\hline \multicolumn{9}{|c|}{ CUD apparent (\%) } \\
\hline Énergie ${ }^{1}$ & $\begin{array}{l}82,5 \\
(2,7)\end{array}$ & $\begin{array}{l}76,0 \\
(1,0)\end{array}$ & $\begin{array}{l}83,8 \\
(0,6)\end{array}$ & $\begin{array}{l}76,4 \\
(2,3)\end{array}$ & $\begin{array}{l}87,1 \\
(1,6)\end{array}$ & $\begin{array}{l}78,4 \\
(2,1)\end{array}$ & $\begin{array}{l}87,0 \\
(0,5)\end{array}$ & $\begin{array}{l}78,1 \\
(0,2)\end{array}$ \\
\hline$M O$ & 85,2 & 79,4 & 86,4 & 80,0 & 89,4 & 81,1 & 89,2 & 81,2 \\
\hline MAT & 83,2 & 76,0 & 84,8 & 76,4 & 87,6 & 79,4 & 86,6 & 77,4 \\
\hline$M G$ & 56,7 & 65,7 & 66,2 & 62,5 & 64,8 & 63,6 & 64,8 & 68,5 \\
\hline$C B$ & 43,2 & 26,2 & 37,4 & 20,7 & 41,8 & 20,1 & 53,5 & 28,2 \\
\hline NDF & 47,4 & 40,4 & 48,1 & 35,0 & 47,9 & 36,1 & 48,8 & 33,4 \\
\hline$A D F$ & 52,3 & 38,9 & 50,2 & 29,2 & 51,0 & 30,5 & 48,0 & 26,5 \\
\hline ENA & 91,1 & 87,8 & 92,2 & 89,0 & 94,3 & 90,7 & 93,0 & 89,1 \\
\hline \multicolumn{9}{|c|}{ Valeurs énergétiques ( $\mathrm{kcal} / \mathrm{kg}$ ) } \\
\hline$E B$ & 3717 & 3913 & 3760 & 3986 & 3751 & 4059 & 3837 & 4048 \\
\hline$E D m$ & $\begin{array}{c}3066 \\
103,1\end{array}$ & $\begin{array}{c}2973 \\
100\end{array}$ & $\begin{array}{l}3151 \\
106,0\end{array}$ & $\begin{array}{c}3045 \\
102,4\end{array}$ & $\begin{array}{c}3268 \\
109,9\end{array}$ & $\begin{array}{l}3182 \\
107,0\end{array}$ & $\begin{array}{c}3339 \\
112,3\end{array}$ & $\begin{array}{c}3163 \\
106,4\end{array}$ \\
\hline$E M^{2}$ & 2911 & 2843 & 2992 & 2906 & 3089 & 3034 & 3188 & 3033 \\
\hline$E M / E D(\%)$ & 95,0 & 95,6 & 95,0 & 95,5 & 94,6 & 95,3 & 95,5 & 95,9 \\
\hline \multirow{2}{*}{$E N E c^{3}$} & 2096 & 2087 & 2182 & 2128 & 2239 & 2191 & 2233 & 2215 \\
\hline & 100,4 & 100 & 104,5 & 101,9 & 107,3 & 105,0 & 107,0 & 106,1 \\
\hline$E N_{6}^{4}$ & 2187 & 2162 & 2289 & 2232 & 2357 & 2302 & 2367 & 2317 \\
\hline$E N_{1}^{4}$ & 2145 & 2115 & 2232 & 2201 & 2326 & 2313 & 2394 & 2298 \\
\hline \multicolumn{9}{|l|}{ Bilan azoté } \\
\hline $\mathrm{N}$ retenu/j $(\mathrm{g})$ & 18,3 & 16,8 & 19,6 & 17,0 & 18,2 & 16,1 & 18,8 & 18,0 \\
\hline CRN 5 & 50,3 & 50,9 & 52,8 & 52,3 & 47,8 & 49,4 & 50,3 & 54,5 \\
\hline
\end{tabular}

1 Entre parenthèses, coefficient de variation (\%). ${ }^{2}$ Énergie métabolisable. ${ }^{3,4}$ Valeurs énergétiques calculées à partir des éléments digestibles $(\mathrm{g} / \mathrm{kg})$ mesurés. ${ }^{3}$ Énergie nette engraissement, calculée à partir des éléments digestibles mesurés, à l'aide de l'équation Nehring et al (1969) figurant au tableau 1. 4 Énergie nette porc (Noblet et al, 1989):

$E N_{6}=2,49 M A D+8,85 M G D+3,54$ amidon $-0,99 C B D+2,83$ résidu $D$

(Résidu $\mathrm{D}=M O D-M A D-M G D-C B D-$ amidon).

$E N_{19}=0,663 E D-0,93 M A T+2,28 M G-1,33 C B+0,76$ amidon, $E D$ en $\mathrm{kcal} / \mathrm{kg}$, quantités d'éléments bruts en $\mathrm{g} /$ kg. ${ }^{5}$ Coefficient de rétention azotée : CRN $(\%)=(\mathrm{N}$ retenu/ $\mathrm{N}$ absorbé $) \times 100$.

contraire surestimée. Une autre explication pourrait résider dans la manifestation d'effets d'interactions digestives négatives, comme on a pu déjà l'enregistrer avec des régimes de même type enrichis simultanément en parois végétales et en matières grasses (Perez et al, 1984 : expérience 21D).

Pour l'ENE, nous avons comparé les valeurs de formulation déduites des tables
(ENEt) et celles calculées à partir des éléments digestibles effectivement mesurés sur les régimes $(E N E C)$. Comme l'indique la figure 3 , la liaison entre ces valeurs est moins étroite $(r=0,71, E T R=2,1 \%)$ qu'entre les valeurs $E D t$ et $E D m$. En effet, l'accroissement de densité énergétique prévu n'a pas été précisément suivi (en particulier avec le régime 8 ) pour les mêmes raisons que précédemment. Mais 


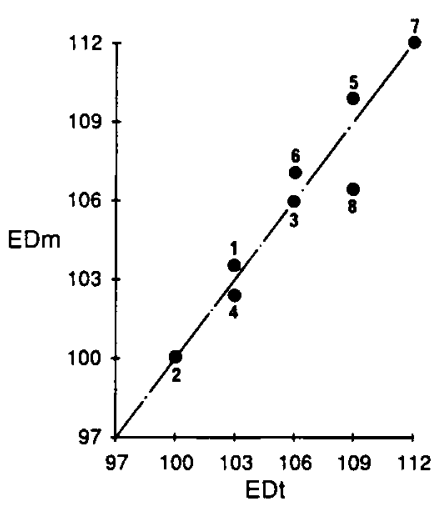

Fig 2. Énergie digestible des régimes : relation entre les valeurs théoriques de formulation $(E D t)$ et les valeurs mesurées $(E D m)$.

surtout, les régimes 3,5 et 7 présentent des valeurs ENE supérieures de $80 \mathrm{kcal} /$ $\mathrm{kg}$ en moyenne à celles des régimes pairs correspondans $(2,4$ et 6$)$. De ces résultats, il découle que la hiérarchie prévue entre les régimes est moins bien respectée pour l'ENE que pour l'ED. Les variations simultanées enregistrées pour les valeurs $E D$ et $E N E$ aboutissent à des

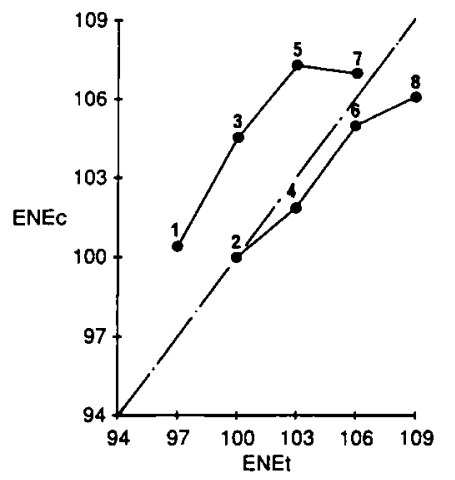

Fig 3. Énergie nette engraissement des régimes : relation entre les valeurs théoriques de formulation (ENEt) et les valeurs calculées à partir des éléments digestibles mesurés (ENEc). densités énergétiques moyennes (les 2 systèmes confondus) conformes aux prévisions pour les couples de régimes 1-2, 23 et 4-5 correspondant aux niveaux théoriques 100,103 et 106 , En revanche, la densité énergétique supérieure escomptée (109) n'a pu être atteinte dans les régimes 7-8 et plafonne au niveau 107.

En outre, nous avons introduit à titre comparatif dans le tableau $V$ les valeurs d'énergie nette porc ( $E N$ porc) selon Noblet et al (1989), utilisant l'équation $n^{\circ} 6$ à partir des éléments digestibles mesurés $\left(E N_{6}\right)$ et l'équation $n^{\circ} 19$ à partir de l'ED mesurée et certains critères de composition chimique : protéines, matières grasses, cellulose brute, amidon ( $E N_{19}$ ).

Enfin, on peut observer que les résultats de bilan azoté ( $\mathrm{N}$ retenu par jour) sont d'une manière générale en faveur des régimes impairs à base de blé et de tourteau de soja, dont la digestibilité des protéines est supérieure à celle observée dans les régimes pairs. Les coefficients de rétention azotée varient peu d'un régime à l'autre dans une zone moyenne de $50-52 \%$.

\section{Résultats de l'expérimentation en lots}

Dans l'essai 2, réalisé en loges collectives, 7 animaux ont été écartés pour des raisons (accident, mortalité) indépendantes de l'expérimentation : les effectifs sont ainsi de 329 animaux, dont 166 femelles et 163 mâles castrés. Les observations de composition corporelle ont été réalisées lors du deuxième abattage, soit sur 130 animaux, dont 78 femelles et 62 mâles castrés.

\section{Performances de croissance}

Les résultats généraux de croissance pour les essais 1 et 2 sont rapportés respective- 


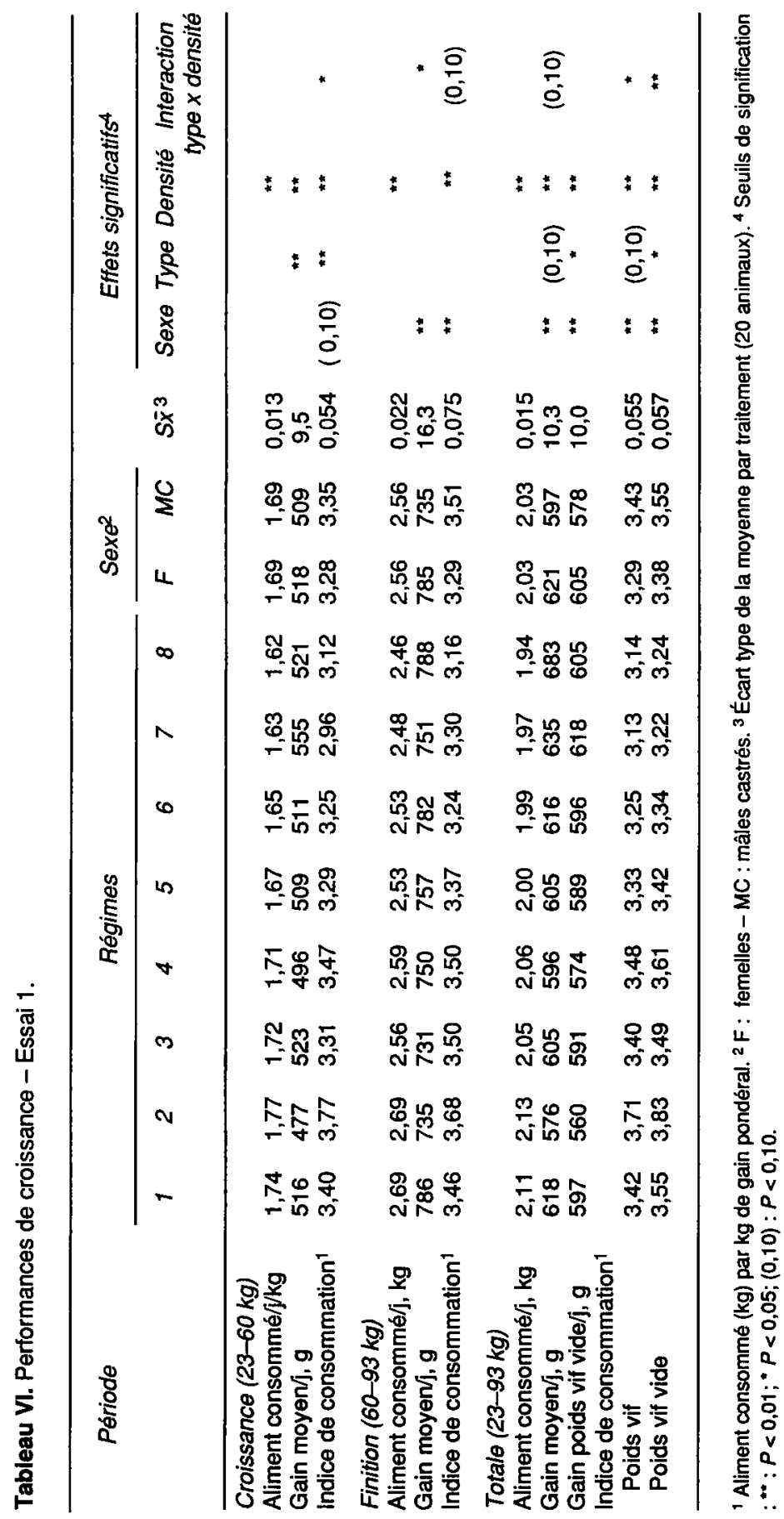




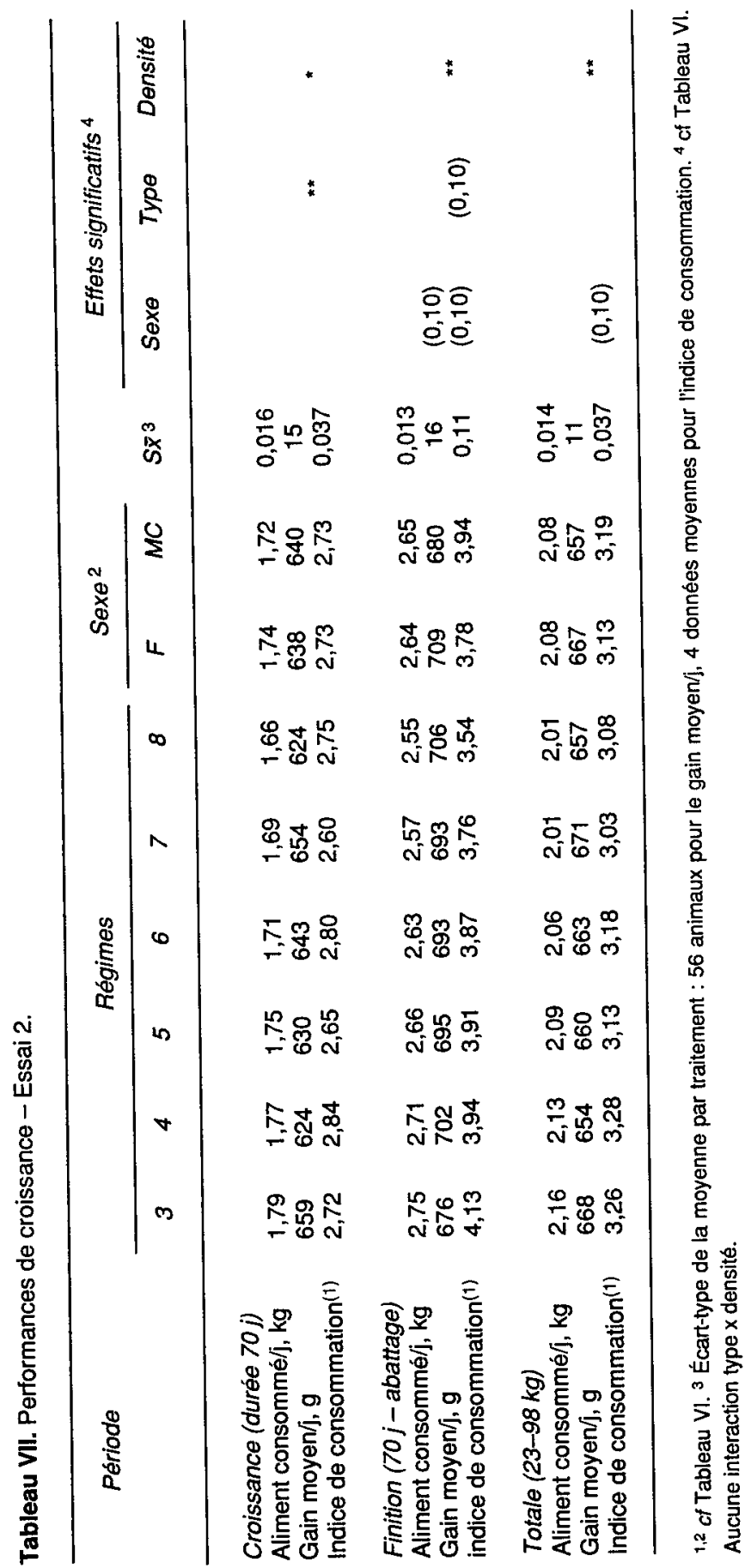


ment dans les tableaux VI et VII. Ils ont été subdivisés en considérant, outre la période totale (début essai-abattage), les 2 périodes intermédiaires :

- "croissance", correspondant à un intervalle de poids constant par animal (en moyenne 23-60 kg) pour l'essai 1 , une durée constante de $70 \mathrm{j}$ dans le cas de l'essai 2;

- "finition", correspondant à l'intervalle de poids constant 60-93 kg pour l'essai 1 et à l'intervalle $70 \mathrm{j}$-abattage pour l'essai 2.

La comparaison des performances des animaux dans les 2 expériences fait apparaître globalement un avantage en faveur de l'essai 2 : $662 \mathrm{~g}$ de gain/j sur la totalité de la période contre $613 \mathrm{~g}$ dans l'essai 1 pour les traitements communs $3-8$, les consommations moyennes journalières d'aliment étant respectivement de 2,08 et $2,00 \mathrm{~kg}$. Cette supériorité, à tempérer en raison du poids moyen d'abattage en moyenne plus élevé dans l'essai 2 (98 kg contre $93 \mathrm{~kg}$ dans l'essai 1), se manifeste principalement au cours de la première période $(639 \mathrm{~g}$ de gain/j contre $519 \mathrm{~g}$ dans l'essai 1 , pour des consommations moyennes respectives d'aliment/j de 1,73 et $1,67 \mathrm{~kg}$ ), bien que l'application d'une durée constante (70 j) dans l'essai 2 ait été à l'avantage des animaux les plus lourds en fin de période. A l'inverse, pendant la période de finition, le niveau de croissance est en moyenne plus élevé dans l'essai 1 que dans l'essai 2 (760 g/j contre 695), probablement en raison d'un effet compensateur consécutivement à une croissance amoindrie en début d'engraissement, et ceci en dépit d'un niveau de consommation moyenne journalière d'aliment plus faible $(2,56 \mathrm{~kg}$ contre 2,65$)$.

Les consommations moyennes d'aliment enregistrées au cours de la période totale d'engraissement respectent les plans d'alimentation du protocole expérimental, en valeur relative par rapport au ni- veau 100 (régimes 5 et 6) : 106 pour les régimes 1 et 2 (essai 1), 103 pour les régimes 3 et 4 (essais 1 et 2), 98 et 97 pour les régimes 7 et 8 , respectivement dans les essais 1 et 2 .

\section{Essai 1}

Dans l'essai 1, on observe des effets hautement significatifs $(P<0,01)$ à la fois de la densité énergétique et du type de régimes (pairs vs impairs) durant la phase initiale de croissance (entre 23 et $60 \mathrm{~kg}$ de poids vif). Au cours de cette période, les meilleures performances sont obtenues avec les régimes à densité énergétique élevée, et les régimes pairs conduisent à des résultats systématiquement plus faibles que leurs homologues impairs ( $\Delta=$ $24 \mathrm{~g} / \mathrm{j})$. En ce qui concerne l'indice de consommation (exprimé en $\mathrm{kg}$ d'aliment par kg de gain), on observe logiquement un effet hautement significatif de la densité énergétique $(P<0,01)$. On relève en outre un effet propre du type de régime $(P<0,01)$ et une interaction significative $(P<0,05)$ type $x$ densité, qui dénotent une détérioration de l'indice de consommation dans les régimes pairs aux faibles densités (fig 4).

Durant la phase de finition (intervalle $60-93 \mathrm{~kg}$ de poids vif), l'interaction type $x$ densité sur le gain pondéral $(P<0,05)$ reflète dans les traitements pairs 6 et 8 une croissance supérieure à celle observée respectivement dans les traitements impairs 5 et 7 , tandis qu'on enregistre un effet de compensation dans le traitement 1 par rapport au traitement 2. Pour l'indice de consommation, outre l'effet densité $(P<$ 0,01 ), le même type d'interaction apparaît au seuil $10 \%$.

Sur l'ensemble de la période d'engraissement l'effet densité $(P<0,01)$ est maintenu sur le gain moyen journalier et sur l'indice de consommation. II en est de même de l'effet type $(P<0,10)$ doublé d'un effet 

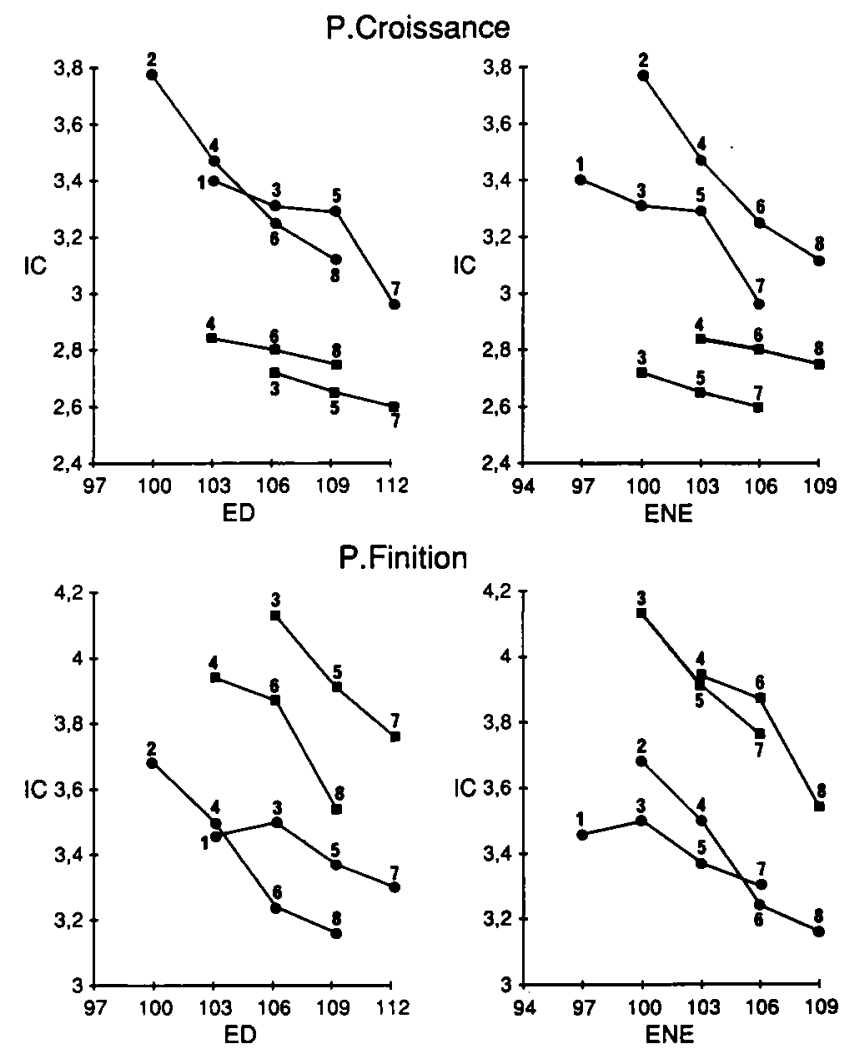

Fig 4. Évolution de lindice de consommation en fonction de la concentration énergétique des régimes selon les systèmes (en valeur relative, par référence à 100 pour le régime 2). essai 1; : essai 2.

d'interaction type $x$ densité, aussi bien pour la vitesse de croissance que pour l'indice de consommation : performances supérieures obtenues avec les régimes pairs aux densités énergétiques élevées, et inversement avec les régimes impairs aux densités énergétiques faibles. II est intéressant de remarquer que la signification des résultats est renforcée avec le gain de poids vif vide ou lorsque l'indice de consommation est exprimé par rapport au poids vif vide, en raison de l'importance des contenus digestifs enregistrés avec les régimes à faible densité énergétique.

\section{Essai 2}

Dans l'essai 2, on n'observe pas l'interaction type $x$ densité qui prévalait dans l'essai 1. En revanche, on retrouve les effets type et densité au niveau à la fois de la vitesse de croissance et de l'indice de consommation (fig 4). Au cours de la première phase de la croissance, l'administration des régimes pairs entraîne dans l'en- 
semble une légère diminution de la vitesse de croissance, se traduisant par un accroissement significatif $(P<0,01)$ de l'indice de consommation. En période de finition, on observe au contraire, avec les régimes pairs, une diminution de l'indice de consommation $(P<0,10)$, ce qui annule l'effet type sur la période totale de croissance.

\section{Composition corporelle}

Le poids moyen de la carcasse chaude était respectivement de 75,0 et $81,0 \mathrm{~kg}$ sur l'ensemble des traitements dans les essais 1 et 2 , pour des poids vifs moyens respectifs à l'abattage de 93 et $97 \mathrm{~kg}$. Dans les 2 essais (tableau VIII et IX), le rendement de la carcasse augmente avec la densité énergétique $(P<0,01)$, tandis qu'un effet type est seulement enregistré dans l'essai $2(P<0,05$ pour le rendement à chaud et $P<0,10$ pour le rendement à froid) : $\Delta=$ 0,6 pour le rendement à chaud en faveur des régimes pairs.

\section{Essai 1}

Dans l'essai 1 , les variations des poids des contenus digestifs, en pourcentage du poids vif, font apparaître des effets type et régime significatifs $(P<0,05)$, les contenus résiduels étant plus importants dans les régimes pairs notamment aux densités énergétiques $2-3$ et 4 . Par contre, le poids des viscères vides diminue à mesure que croît la densité énergétique, en relation avec le niveau d'alimentation. En dehors de l'effet sexe (femelles vs mâles castrés) habituellement observé, on constate une diminution de la longueur restreinte de la carcasse aux densités énergétiques les plus élevées $(P<0,05)$. Parmi les mesures linéaires de gras et de maigre, réalisées au FOM, on enregistre une interaction type $x$ densité au seuil $5 \%$ sur l'épaisseur de maigre $\left(X_{5}^{\prime}\right)$ : cette dernière est plus réduite dans les régimes pairs aux faibles densités énergétiques, l'inverse étant constaté aux densités énergétiques élevées. De même, le pourcentage de muscle, obtenu à partir des résultats de la découpe DPN, est plus élevé dans les régimes pairs aux fortes densités énergétiques ( 8 vs 7 et 6 vs 5). L'évolution inverse est observée pour le pourcentage de gras (effet type significatif au seuil 10\%).

L'analyse des résultats de la découpe et de la dissection du jambon fait apparaître certaines différences dans la distribution des tissus maigres et gras dans la carcasse, selon le type de régime et la densité énergétique. Ainsi, après analyse de covariance à poids de . demi-carcasse découpée égal, on observe un effet type significatif $(P<0,01)$ sur le poids de longe, plus marqué avec les régimes pairs notamment aux fortes densités énergétiques. II en résulte un effet type au seuil $10 \%$ sur le rapport longe/bardière, tandis que le poids de poitrine a tendance à être plus faible $(P<0,10)$ dans les régimes pairs aux densités énergétiques élevées. L'accroissement de la densité énergétique est associé à une augmentation du poids du jambon $(P<0,05)$ et à une diminution du poids de la panne $(P<0,05)$. En comparant les résultats de la dissection du jambon, on constate qu'aux densités énergétiques faibles le pourcentage d'os est significativement plus élevé avec les régimes pairs, tandis que la tendance inverse apparaît aux fortes densités énergétiques (effet régime au seuil 5\%). En ce qui concerne les dépôts de tissus estimés à partir des résultats de la découpe (DPN), les effets densité $(P<0,01)$ et type $x$ densité $(P<0,05)$ sur le gain de muscle traduisent surtout des valeurs abaissées dans les régimes pairs aux densités énergétiques faibles (2 vs 1 et 4 vs 3), tandis que les dépôts gras apparaissent réduits dans les régimes 


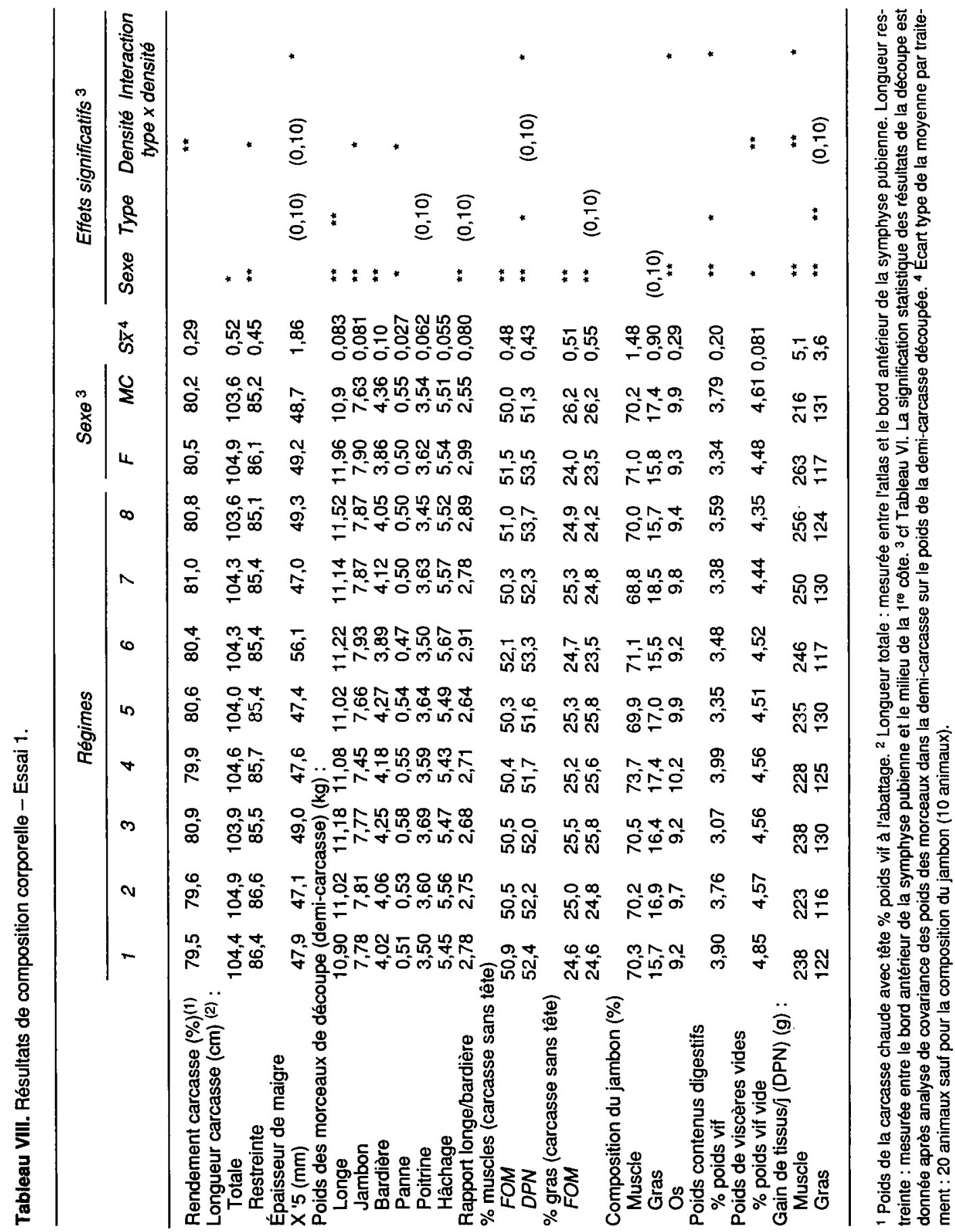




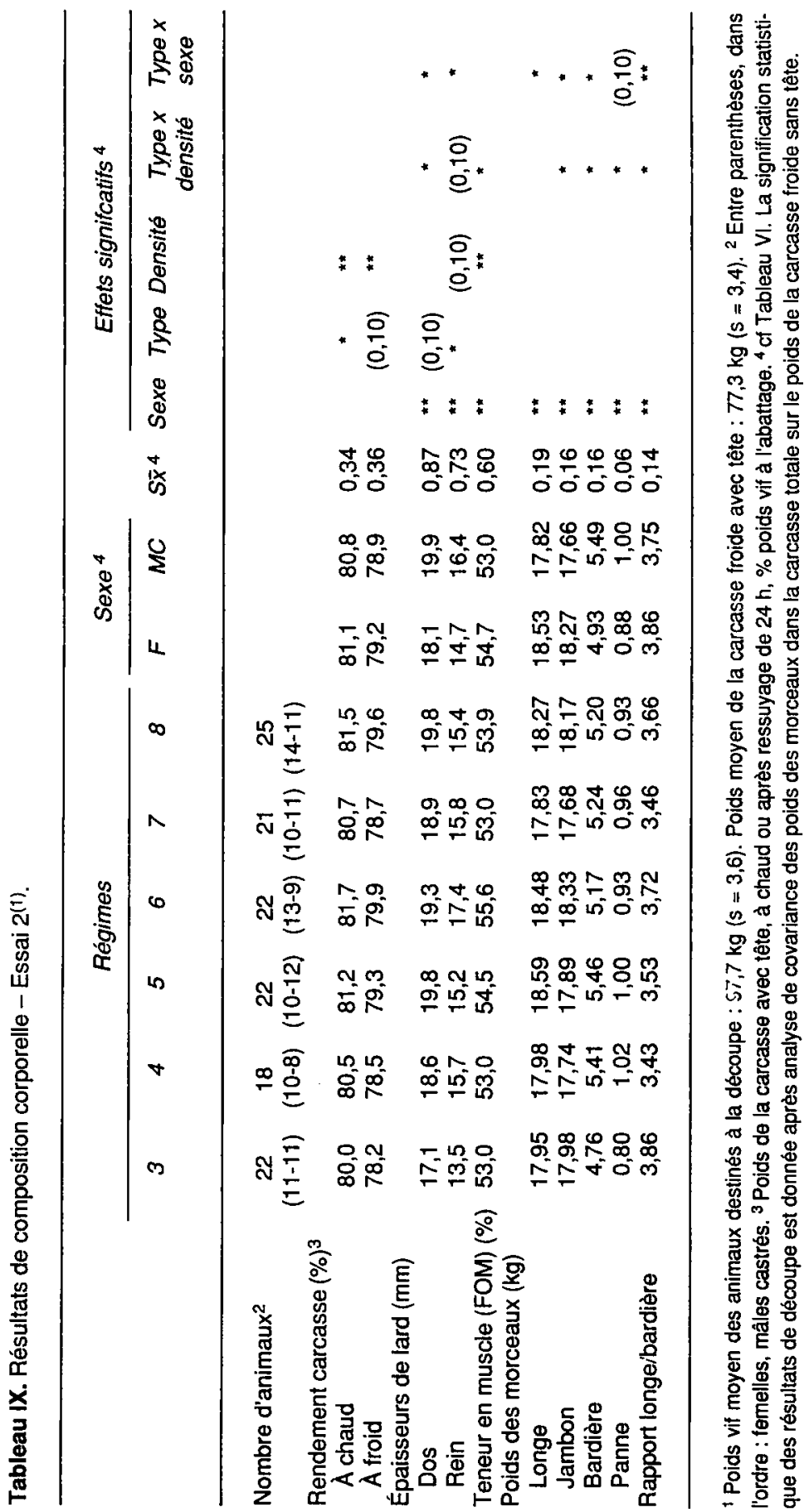


pairs comparativement aux régimes impairs.

\section{Essai 2}

Dans l'essai 2, seules les épaisseurs de lard au dos et au rein sont modifiées globalement par le type et la densité énergétique : augmentation de l'épaisseur au dos dans les régimes pairs $(P<0,10)$; même effet du type sur l'épaisseur au rein $(P<0,05)$, et augmentation de cette dernière avec l'accroissement de la densité énergétique $(P<0,10)$. Corrélativement, on note un effet densité $(P<0,01)$ sur la teneur en muscle (FOM) : les valeurs les plus élevées sont obtenues avec la densité énergétique intermédiaire (régimes 5 et 6). Après analyse de covariance sur les poids des morceaux de découpe en fonction du poids de la carcasse froide, on remarque une interaction type $x$ densité $(P<$ $0,05)$ pour le jambon, la bardière, la panne et le rapport longe/bardière : augmentation de l'importance des morceaux maigres et diminution de l'adiposité des carcasses dans les régimes pairs aux densités énergétiques élevées ( 8 vs 7 et 6 vs 5) et l'inverse à la densité énergétique la plus faible (4 vs 3). Mais, contrairement à l'essai 1, dans lequel on ne relevait aucune interaction entre le sexe et les facteurs expérimentaux, l'examen des résultats de composition corporelle fait ressortir une série d'interactions type $x$ sexe pour plusieurs des critères considérés : épaisseurs du lard au dos et au rein, poids ajustés de longe, jambon, bardière et panne, rapport longe/bardière. D'une manière générale, dans la population d'animaux croisés utilisés dans cette expérience, les femelles réagissent plus favorablement que les mâles castrés aux régimes pairs aux plus fortes densités énergétiques (2-3 et 4) par une augmentation de l'importance des masses maigres (longe, jambon) au détriment du gras (épaisseurs de lard, bar- dière, panne), ce qui explique les variations du rapport longe/bardière.

De la comparaison des caractéristiques de composition corporelle d'une expérience à l'autre il ressort globalement que les animaux de l'essai 2 sont plus maigres que ceux de l'essai 1.

\section{Incidence du choix du systeme et du mode de calcul de la valeur énergétique sur l'efficacité énergétique, la prévision des dépôts tissulaires et de l'énergie fixée}

Pour évaluer l'efficacité énergétique selon les systèmes, nous avons exprimé l'indice de consommation en quantité d'énergie (Mcal) par kg de gain de poids vif (essais 1 et 2) ou poids vif vide (essai 1) suivant les différentes modalités définies précédemment : EDt, EDm, ENEt, ENEc, EN 6 et $E N_{19}$

A l'examen des résultats de l'essai 1 (tableau $X$ ), on enregistre des effets densité et type $x$ densité pour l'ensemble des estimations. L'effet type apparaît seulement lorsque l'indice de consommation est exprimé en valeur ENEt. Les mêmes constatations sont faites lorsque l'indice de consommation est calculé par rapport au gain de poids vif vide. Dans l'essai 2 (tableau $\mathrm{XI}$ ), on retrouve l'effet type avec l'ENEt de même qu'avec l'EDm. L'effet densité atteint le seuil de signification pour l'ENEc $(P<0,05)$, ainsi que pour l'EN $(P<0,05)$, mais, en l'absence des traitements extrêmes 1 et 2, on n'observe plus d'interaction type $x$ densité. Enfin, si l'on considère l'EDt déduite des tables et l'EN 19 , il n'apparaît plus d'effet lié au régime.

Afin de tenir compte des effets dépressifs des régimes extrêmes (notamment des régimes pairs 2 et 4 ) sur les performances de croissance, nous avons procédé, dans l'essai 1, à une analyse de cova- 


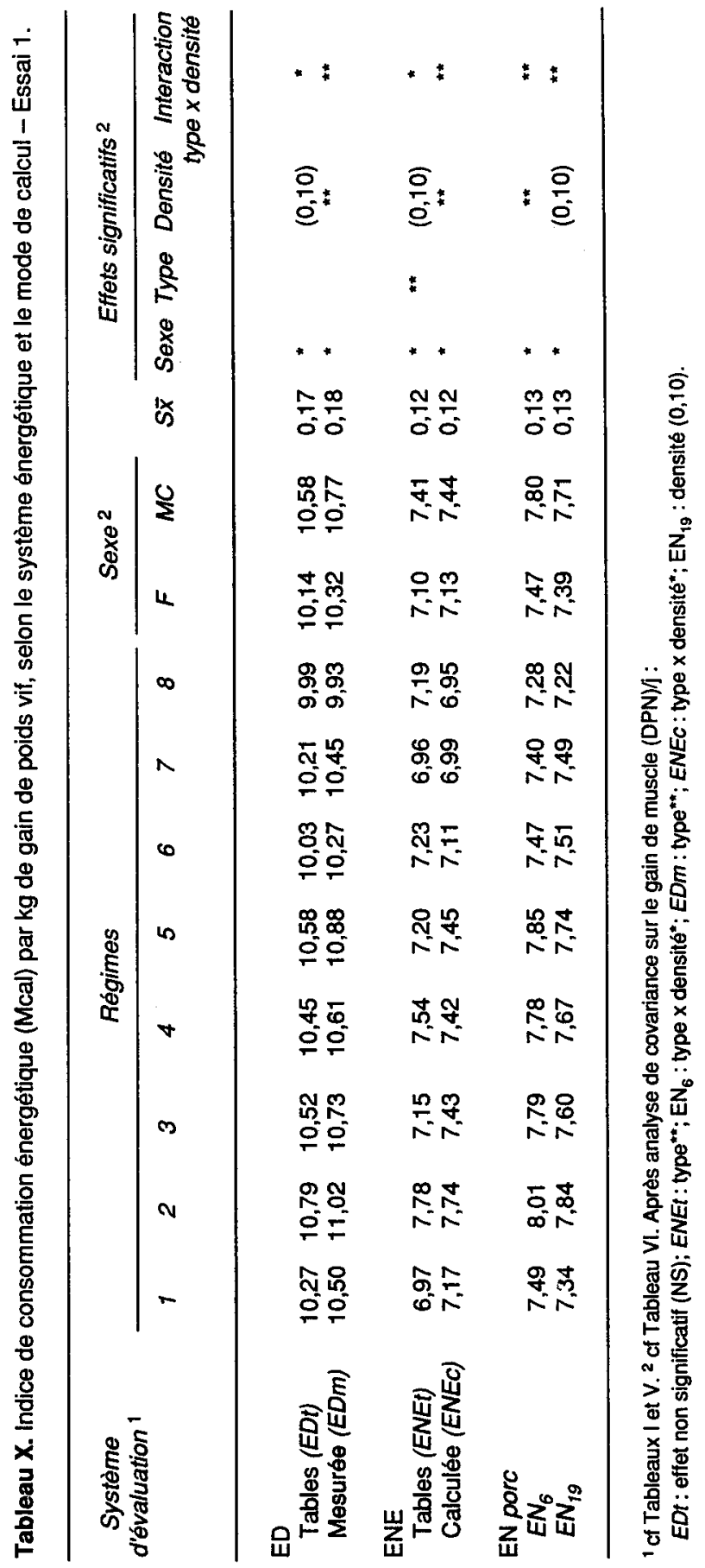




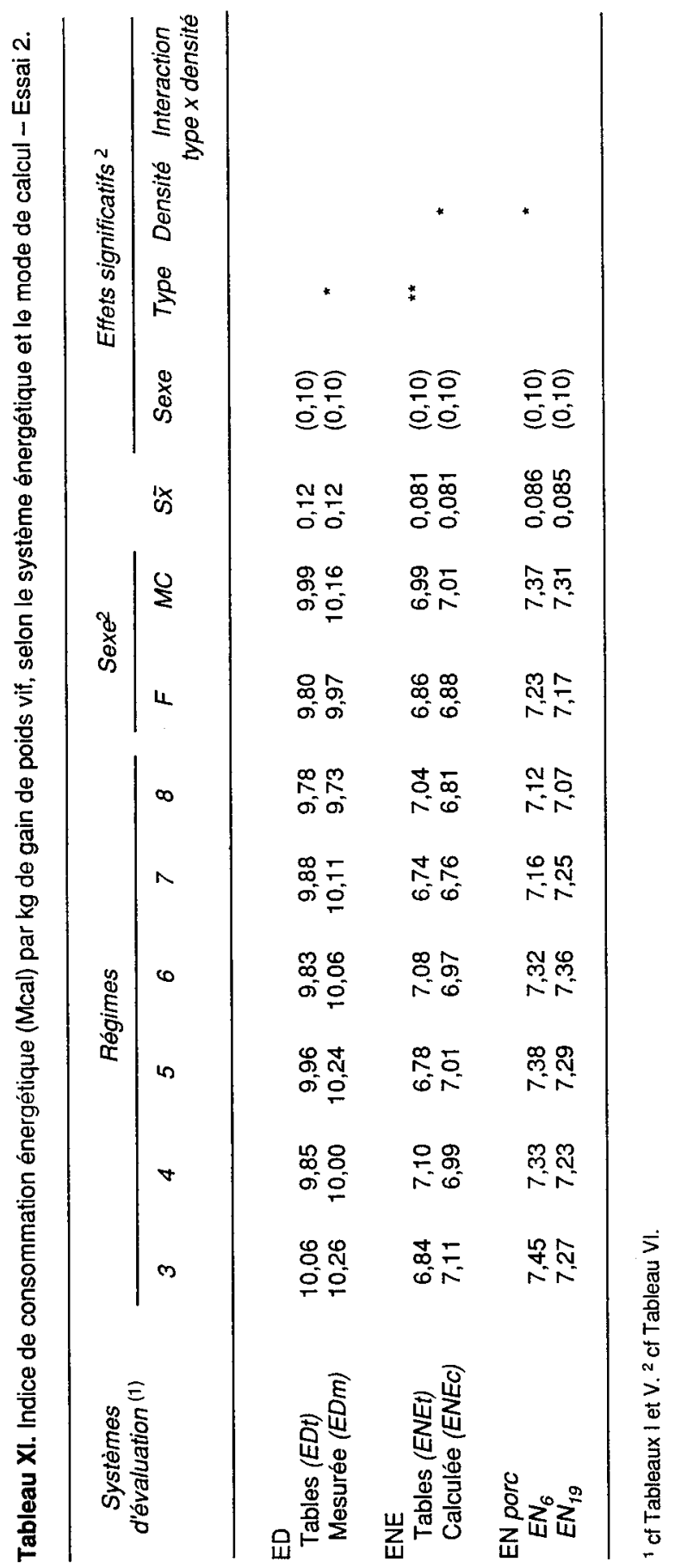


riance des données d'indice de consommation, suivant les systèmes, sur le gain journalier de muscle. Lorsque l'indice de consommation est rapporté au gain de poids vif vide, les valeurs exprimées dans le système $E N$ porc (équations nos 6 et 19: Noblet et al, 1989) sont indépendantes des traitements expérimentaux, contrairement à ce qui est observé avec l'ED mesurée (effet type au seuil $5 \%$ ) et avec le système ENE (effet type au seuil $1 \%$ pour les valeurs déduites des tables, interaction type $x$ densité au seuil $10 \%$ pour les valeurs corrigées en fonction des résultats de digestibilité).

En outre, à partir des résultats de l'essai 1 , nous avons procédé à une analyse de régression de la consommation moyenne journalière d'énergie sur le gain journalier de muscle et de gras d'une part, et la quantité d'énergie fixée par jour d'autre part, cette dernière étant estimée à partir de l'énergie des dépôts tissulaires (tableau XII). Qu'il s'agisse des gains de tissus ou de l'énergie fixée, le choix du système $E N E$, comparativement à l'ED et à I'EN porc, conduit à des valeurs plus faibles des corrélations et à un écart type résiduel plus important. Ces résultats sont à nuancer en fonction du mode de calcul, selon que l'on se base sur les estimations initiales ou les valeurs de digestibilité effectivement mesurées. Ainsi, on peut remarquer un gain de précision avec les résultats de digestibilité dans le cas de l'ENE.

\section{DISCUSSION}

L'objectif de cette étude était de comparer 2 systèmes d'évaluation énergétique des aliments (énergie nette engraissement, énergie digestible) pour la prévision des performances de production du porc, notamment sur la base de l'efficacité alimen- taire globable et des dépôts respectifs de muscle et de gras, afin de tenir compte des différences de partition de l'énergie fixée. Les aliments étudiés étaient formulés et les plans de rationnement fixés de manière à fournir la même quantité journalière d'énergie et de lysine. Dans ces conditions, tous les régimes étaient censés procurer le même niveau de performances en termes de gain journalier de muscle et de dépôt gras, avec une valeur d'indice de consommation énergétique (Mcal par $\mathrm{kg}$ de gain pondéral) sensiblement constante et indépendante de leurs caractéristiques de composition (type, densité énergétique et interaction).

Jusqu'à présent très peu d'études ont été réalisées afin d'évaluer l'efficacité d'un système énergétique pour la prévision des performances de production (voir Henry et Perez, 1982). Les travaux de Borggreve et al (1975) ont fait apparaître les défauts du système ENE en montrant que pour un même apport énergétique dans ce système, les variations d'indice de consommation pouvaient être attribuées aux différences de teneurs en amidon des régimes. Plus récemment Jongbloed et al (1986), en formulant dans le système ENE, ont obtenu de meilleures performances avec des régimes essentiellement à base de céréales comparativement à des régimes riches en produits de substitution. De la même façon, nous avons constaté la supériorité de régimes simplifiés à base de céréales sur des régimes complexes pour une même concentration en énergie digestible (Chauvel et al, 1986). A partir de données complémentaires de digestibilité des matières grasses et des constituants pariétaux sur ces mêmes régimes (JM Perez, résultats non publiés), nous avons estimé leurs valeurs en énergie nette et calculé les indices de conversion énergétique correspondants. Les résultats indiquent que l'ENE n'est pas un prédicteur plus précis des performances de croissance que l'ED. 


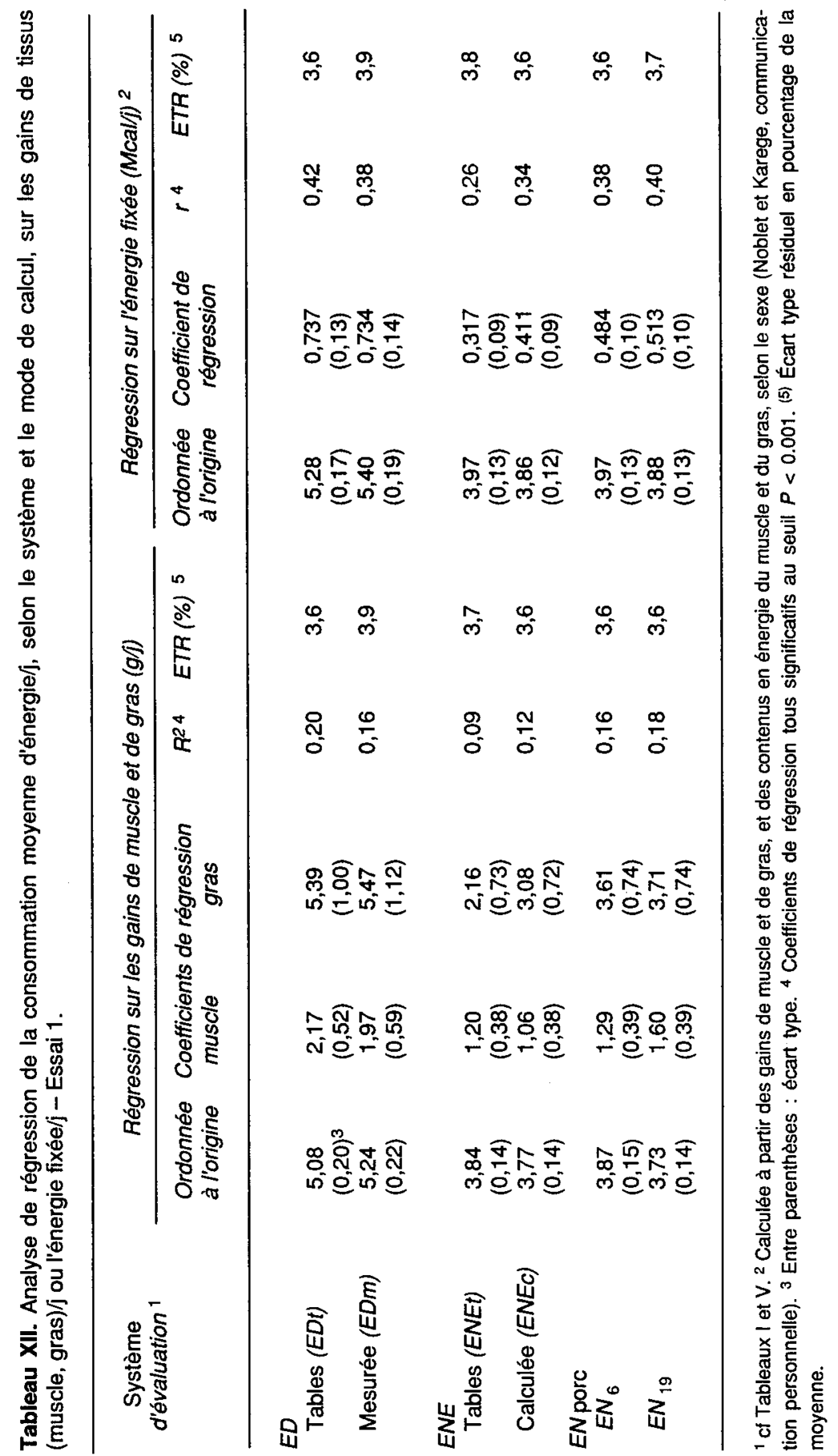


Dans notre étude, la comparaison des systèmes $E D$ et $E N E$ a été effectuée à partir d'un ensemble de 8 régimes choisis en élargissant au maximum la plage de variation des caractéristiques nutritionnelles. C'est ainsi que les 4 régimes «impairs", à base de blé et à teneur relativement élevée en protéines, induits par la formulation dans le système $E D$, se sont trouvé opposés aux 4 régimes "pairs", à base d'orge, moins riches en protéines et bénéficiant d'une incorporation plus importante de matières grasses et de produits de substitution, comme le favorise la formulation dans le système ENE. Ce faisant, les régimes pairs ont présenté des teneurs suboptimales en certains acides aminés (thréonine, méthionine) dont le caractère limitant a été accentué par la faible digestibilité apparente des protéines mesurée dans ces régimes. Ce risque de déséquilibre a été discuté antérieurement dans le cas de l'utilisation de régimes composés de céréales, à teneurs abaissées en protéines après supplémentation en lysine (Henry, Perez, 1986). La distinction des régimes selon le type (impairs vs pairs) est ainsi le reflet de la formulation dans l'un ou l'autre des 2 systèmes ED ou ENE. Elle se traduit, au plan des performances dans les 2 essais, par un effet du type de régime au cours de la première phase de la croissance. Les valeurs plus faibles du gain moyen pondéral et du gain de muscle, comme de l'efficacité alimentaire, enregistrées chez les animaux recevant les régimes pairs principalement aux faibles densités énergétiques (régimes 2 et 4 ) sont à relier à une teneur insuffisante en acides aminés limitants secondaires (thréonine et/ou méthionine). Ceci explique que l'analyse de covariance, dans l'essai 1 , de l'indice de consommation énergétique sur le gain journalier de muscle permet d'atténuer, voire d'annuler, les effets du type et de la densité énergétique dans la prévision des performances de production. Par ailleurs, les faibles niveaux de densité énergétique (régimes 1 à 4) ont entraîné, en raison de l'accroissement de la teneur en parois végétales, une augmentation de poids des viscères et corrélativement une réduction du rendement à l'abattage. Cela explique que dans l'essai 1 l'expression de l'indice de consommation énergétique sur la base du gain de poids vif vide conduit à atténuer les effets des facteurs alimentaires.

Les résultats des 2 essais ont permis de dégager pour la plupart des critères de croissance et de composition corporelle une interaction type $x$ densité énergétique significative. Ce sont les régimes pairs aux densités énergétiques élevées (6 et 8 ) qui permettent d'obtenir les carcasses les plus maigres (pourcentage et gain de muscle, poids de longe et de jambon), tandis que les critères d'adiposité sont affectés négativement (dépôt journalier de gras, poids de poitrine et de panne). L'amélioration de la croissance et de l'efficacité alimentaire avec ce type de régime est surtout marquée en période de finition. Cela dénote l'influence favorable de la formulation dans le système ENE au bénéfice des régimes à forte densité en énergie nette (incorporation plus élevée de matières grasses et teneur plus faible en protéines) comparativement au système $E D$, alors qu'en début de croissance le risque était plus grand de faire apparaître des apports sublimitants en certains acides aminés pour la croissance musculaire. Cet effet favorable semble renforcé dans l'essai 2 , comparativement à l'essai 1 , où les animaux ont été abattus à un poids plus léger.

II est intéressant de noter que seul l'essai 2, dans lequel on utilisait des animaux d'un niveau génétique supérieur à celui de l'essai 1 (porcs croisés au lieu d'animaux Large White), a révélé une interaction significative entre le sexe et le type de régime sur les caractéristiques de composition corporelle, l'influence favorable 
des régimes pairs aux densités énergétiques élevées ( 6 et 8 ) sur la teneur en tissus maigres étant plus marquée chez les femelles que chez les mâles castrés. Cette différence de réponse selon le type sexuel peut être attribuée, comme l'ont souligné Desmoulin et Bonneau (1979), à l'effet dépressif de la castration du mâle sur la pleine expression des potentialités génétiques pour la croissance musculaire.

L'originalité de notre approche a été d'établir une comparaison de 2 systèmes d'évaluation énergétique ( $E D, E N E)$ pour la prévision des performances du porc par le biais d'une densité énergétique moyenne traduisant les variations simultanées des concentrations en énergie des régimes dans chacun des 2 systèmes. Globalement, si on considère l'incidence du système sur les variations de l'indice de consommation énergétique (tableaux $X$ et XI) ou sur la prévision des dépôts tissulaires et de l'énergie fixée (tableau XII), les performances de production semblent mieux en relation avec l'ED qu'avec l'ENE. Cela provient notamment du fait que ce dernier système, en privilégiant la formulation des régimes de type pair, a pu induire un équilibre en acides aminés moins favorable. II convient de noter cependant que les régimes ont été formulés sur la base des teneurs en acides aminés totaux : la prise en compte, lors de la formulation, des teneurs en acides aminés digestibles au niveau iléal (sur une base encore à définir) aurait sans doute permis d'atténuer cette différence. En réalité, dans la présente étude, les systèmes $E D$ et $E N E$ ont été comparés dans leur globalité, en intégrant notamment les facteurs de risque liés au choix de certaines matières premières, compte tenu de leur prix d'intérêt dans le système considéré. La formulation de régimes présentant des caractéristiques de composition nettement différenciées, a conduit, par le risque d'apports sublimitants de certaines acides aminés (méthionine et thréonine en particulier), à des performances amoindries en début d'essai, ce qui explique le niveau relativement modeste des résultats observés au cours de cette phase expérimentale, principalement dans l'essai 1.

En outre, les travaux récents de Noblet et al $(1989,1990)$ sur l'estimation de la valeur en énergie nette des aliments (EN porc) nous ont permis de prendre en compte ce nouveau système pour la prévision des performances de production. Comparativement au système ENE, l'EN porc présente l'avantage de mieux discriminer les valeurs d'utilisation des nutriments, notamment en privilégiant l'amidon par rapport aux parois digestibles. Ceci explique en particulier que dans notre étude I'EN porc permet d'assurer la meilleure constance de l'indice de consommation énergétique (Mcal par $\mathrm{kg}$ de gain de poids vif ou vide) indépendamment des facteurs nutritionnels considérés (type de régime, densité énergétique). De même, pour la prédiction des gains de tissus (muscle et dépôts gras) ou de l'énergie fixée, le système EN porc se révèle globablement plus précis que le système ENE. II reste à préciser les mérites comparés des 2 systèmes sur le plan de l'utilisation stricte de l'énergie alimentaire pour la croissance du porc. Un travail en cours (Noblet et al, résultats non publiés) permettra de conclure sur ce point.

\section{CONCLUSION}

En définitive, les résultats de notre étude ont fait apparaître la complexité de la comparaison des systèmes d'évaluation énergétique des aliments pour la prévision des performances de croissance du porc. En dehors de l'utilisation de l'énergie propre- 
ment dite, cette comparaison intègre en effet des aspects liés à la fois au choix des matières premières et aux marges de sécurité consenties lors de la formulation des régimes. C'est pourquoi, les conclusions qui s'en dégagent sont à nuancer en fonction de la stratégie de formulation propre à chacun des systèmes d'évaluation énergétique.

\section{REMERCIEMENTS}

Cette étude a été soutenue financièrement par le ministère de la Recherche et de la Technologie (décision d'aide 84.G.0833), dans le cadre de l'action concertée "Protéines en alimentation animale". Nous adressons nos sincères remerciements à l'ensemble du personnel des installations expérimentales, laboratoire et secrétariat de l'INRA, de Sanders Aliments (Sourches, Le Mans) et de l'INA Paris-Grignon qui ont contribué à la réalisation de ce travail.

\section{RÉFÉRENCES}

Borggreve CJ, Van Kempen GJM, Cornelissen JP, Grimbergen AHM (1975) The net energy content of pig feeds according to the Rostock formula. The value of starch in the feed. $Z$ Tierphysiol Tierernähr Futtermittelk 34, 199204

Brette C, Duquenne C, Henry Y, Jacquot L, Palisse-Roussel, Perez JM, Sauvant D, Theillaud V (1986) Influence du choix du système énergétique sur les résultats de la formulation des aliments pour les porcs en croissance. Journ Rech Porcine Fr 18, 91102

Centraal Veevoederbureau (CVB) (1983) Veevoedertabel. In: Gegevens over Voederwaarde, Verteerbaarheid en Samenstelling. CVB Lelystad, Pays-Bas, C1-C11

Chauvel J, Gatel F, Grosjean F, Latimier P, Le Meur D, Perez JM, Saulnier J (1986) Utilisation comparée de quatre régimes isoénergétiques par le porc à l'engrais : résul- tats d'un essai concerté ITP-ITCF-EDEINRA. Journ Rech Porcine Fr 18, 103-110

Desmoulin B, Bonneau M (1979) Production des viandes de porcs mâles entiers ou castrés chez les types Piétrain ou Landrace Belge. Journ Rech Porcine Fr 11, 113-120

Desmoulin B, Ecolan P, Bonneau M (1988) Estimation de la composition tissulaire des carcasses de porcs : récapitulatif de diverses méthodes utilisables en expérimentation. INRA Prod Anim 1, 59-64

Giger S, Sauvant D, Dorléans M, Morand-Fehr (1979) Détermination semi-automatique des constituants membranaires des aliments concentrés par la méthode de Van Soest. 30th Annual Meeting of the EAAP, Harrogate, England, July 1979. Commission on Animal Feeding, N3.5

Henry Y, Perez JM (1982) Les systèmes d'évaluation de l'énergie dans l'alimentation du porc (1). Doss Elev 5 (1), 51-66

Henry Y, Perez JM (1983) Les systèmes d'évaluation de l'énergie dans l'alimentation du porc (2). Doss Elev 5 (2), 49-64

Henry Y, Perez JM (1986) Effets de la supplémentation en lysine, associée à une réduction du taux de protéines, dans des régimes à base de maîs ou de blé sur les performances de croissance du porc. Journ Rech Porcine Fr 18, 57-66

Henry Y, Vogt H, Zoiopoulos PE (1988) Livestock feed resources and feed evaluation in Europe. Present situation and future prospects. III. 4. Pigs and Poultry. Livest Prod Sci 19, 299-354

INRA (1984) L'Alimentation des animaux monogastriques : porc, lapin, volailles. INRA, Paris, $282 \mathrm{p}$

Jongbloed AW, Van Diepen JTM, Smits B (1986) Het effect van rantsoenen met veel granen of bijprodukten op de technische resultaten bij mestvarkens. Rapport IVVO 176, Lelystad, Pays-Bas

Nehring K, Schiemann R, Hoffmann L (1969) A new system for energetic evaluation of food on the basis of net energy for fattening. In : Energy Metabolism of Farm Animals (Blaxter K, Kielanowski J, Thorbek G, eds) EAAP Publ No 12, Oriel Press, Newcastle, 41-50 
Noblet J, Fortune $H$, Dubois $S$, Henry $Y$ (1989) Nouvelles Bases d'estimation des teneurs en énergie digestible, métabolisable et nette des aliments pour le porc. INRA, Paris, $106 \mathrm{p}$

Noblet J, Fortune H, Dupire C, Dubois S (1990) Valeur nutritonnelle de treize matières premières pour le porc en croissance. 1. Teneurs en énergie digestible, métabolisable et nette. Conséquences du choix du système énergétique. Journ Rech Porcine F 22, 175184

Perez JM, Ramihone R, Henry Y (1984) Prédiction de la valeur énergétique des aliments composés destinés au porc : étude expérimentale. INRA, Paris, $95 \mathrm{p}$
SAS Institute Inc (1988) SAS/STAT Guide for Personal Computers, Version 6. Cary, NC : SAS (Statistical Analysis system) institute Inc, $1028 \mathrm{p}$

Schiemann R, Nehring $K$, Hoffmann L, Jentsch W, Chudy A (1971) Energetische Futterbewertung und Energienormen. VEB Deutscher Landwirtschaftsverlag, Berlin, $344 p$

Van Soest PJ (1963) Use of detergents in the analysis of fibrous feeds. II. A rapid method of determination of cellulose and lignin. $J$ Assoc Off Agric Chem 46, 829-835

Van Soest PJ, Wine RH (1967) Use of detergents in the analysis of fibrous feeds. IV. Determination of plant cell-wall constituents. $J$ Assoc Off Agric Chem 50, 50-55 